\title{
Spin-orbit-induced longitudinal spin-polarized currents in nonmagnetic solids
}

\author{
S. Wimmer, ${ }^{*}$ M. Seemann, K. Chadova, D. Ködderitzsch, ${ }^{\dagger}$ and H. Ebert \\ Department Chemie/Physikalische Chemie, Ludwig-Maximilians-Universität München, Butenandtstrasse 11, 81377 München, Germany
}

(Received 26 November 2014; revised manuscript received 9 June 2015; published 2 July 2015)

\begin{abstract}
For certain nonmagnetic solids with low symmetry the occurrence of spin-polarized longitudinal currents is predicted. These arise due to an interplay of spin-orbit interaction and the particular crystal symmetry. This result is derived using a group-theoretical scheme that allows investigating the symmetry properties of any linear response tensor relevant to the field of spintronics. For the spin conductivity tensor it is shown that only the magnetic Laue group has to be considered in this context. Within the introduced general scheme also the spin Hall and additional related transverse effects emerge without making reference to the two-current model. Numerical studies confirm these findings and demonstrate for $\left(\mathrm{Au}_{1-x} \mathrm{Pt}_{x}\right)_{4} \mathrm{Sc}$ that the longitudinal spin conductivity may be on the same order of magnitude as the conventional transverse one. The presented formalism only relies on the magnetic space group and therefore is universally applicable to any type of magnetic order.
\end{abstract}

DOI: 10.1103/PhysRevB.92.041101

PACS number(s): 72.25.Ba, 61.50.Ah, 71.15.Rf, 72.15.Qm

The discovery of the spin Hall effect [1-3] (SHE) with its particular feature of converting a longitudinal charge current into a transverse spin current has sparked numerous studies that finally led to a deep understanding of many effects that are spin-orbit induced. Among them are the enigmatic anomalous Hall effect (AHE) that shares the same origin as the SHE and many new phenomena emerging from a coupling of spin, charge, and orbital degrees of freedom in electric fields as well as temperature gradients. Examples of these are the Edelstein effect (EE [4,5]) and the spincaloritronic pendants to the SHE and AHE, namely the spin and anomalous Nernst effects (SNE [6,7], ANE [8,9]), respectively. Many models have been formulated that aim to capture particular contributions to theses effects. For instance, the concept of the semiclassical Berry phase that can be determined on the basis of the band structure of perfect crystalline systems is connected to so called intrinsic contributions [10-12]. Extrinsic contributions arising from scattering at impurities in nonperfect systems can, for example, be obtained from diagrammatic methods [13] or Boltzmann transport theory [14].

The aforementioned transport phenomena and their different contributions being linear in the driving fields should, in principle, be described using the fundamental Kubo formula for the corresponding response function [15],

$$
\tau_{i j}(\omega)=\int_{0}^{\infty} d t e^{-i \omega t} \int_{0}^{\beta} d \lambda \operatorname{Tr}\left[\rho \hat{A}_{j} \hat{B}_{i}(t+i \hbar \lambda)\right] .
$$

The effects then emerge from the characteristics of the underlying Hamiltonian, the pair of chosen operators for perturbation $\left(\hat{A}_{j}\right)$ and observable $\left(\hat{B}_{i}\right)$, and the symmetry of the system. Due to the intractability of the problem to exactly solve the Kubo formula for a realistic system in practice one has to resort to approximations and/or models. However, irrespective of this problem one can still analyze the transformation properties of response tensors $\underline{\tau}$ determined by the Kubo formula to make statements about which effects are in principle allowed, i.e., which nonvanishing tensor elements

\footnotetext{
*sebastian.wimmer@cup.uni-muenchen.de

†diemo.koedderitzsch@cup.uni-muenchen.de
}

may occur given a particular transformation property of the operators appearing in Eq. (1). This route has been followed by Kleiner [15,16], who demonstrated that the occurrence of the AHE is predicted by such a space-time symmetry analysis. Furthermore, considering in addition heat currents he derived general Onsager reciprocity relations.

Here, by extending this approach and applying it in the context of spin current operators [17] we demonstrate that in certain nonmagnetic low-symmetry systems an electric field can induce a longitudinal spin-polarized current [18] that has hitherto evaded perception, and complements the transverse spin Hall effect. Furthermore two additional transverse effects are found which differ from the SHE by the direction of polarization. The results of the group-theoretical analysis are independently verified for an alloy bulk system performing relativistic first-principles Kubo-type transport calculations. The presented formalism is furthermore very general, because (i) it allows identifying nontrivial response phenomena as nonzero elements in respective response tensors, as, e.g., the AHE, (ii) it applies to both magnetic and nonmagnetic systems, and (iii) it is free of the notion of a two-current model often used as an approximation in discussing spintronic phenomena; instead it is based on the concept of spin (polarization) current densities.

The material-specific features of any transport property may be discussed on the basis of the corresponding response function tensor $\boldsymbol{\tau}$. Concerning this, the shape of the tensor $\underline{\tau}$, i.e., the occurrence and degeneracy of nonzero elements, reflecting the symmetry of the investigated solid, is obviously of central importance. To find, in particular, the shape of the spin conductivity tensor, Kleiner's scheme [15] to deal with the symmetry properties of ordinary transport tensors has been extended to the case when the response observable is represented by an arbitrary operator product of the form $\left(\hat{B}_{i} \hat{C}_{j}\right)$ while an operator $\hat{A}_{k}$ represents the perturbation and the operators $\hat{A}_{k}, \hat{B}_{j}$, and $\hat{C}_{i}$ are seen as the Cartesian components of vector operators. Within Kubo's linear response formalism the corresponding frequency- $(\omega)$-dependent response function is then given by

$$
\begin{aligned}
\tau_{\left(\hat{B}_{i} \hat{C}_{j}\right) \hat{A}_{k}}(\omega, \mathbf{H})= & \int_{0}^{\infty} d t e^{-i \omega t} \int_{0}^{\beta} d \lambda \operatorname{Tr}\left[\rho(\mathbf{H}) \hat{A}_{k}\right. \\
& \left.\times \hat{B}_{i}(t+i \hbar \lambda ; \mathbf{H}) \hat{C}_{j}(t+i \hbar \lambda ; \mathbf{H})\right],
\end{aligned}
$$


TABLE I. Electrical $(\underline{\boldsymbol{\sigma}})$ and spin $\left(\underline{\boldsymbol{\sigma}}^{k}\right)$ conductivity tensor forms for the magnetic Laue groups discussed in the text $[18,19]$. Below each group symbol an example for a material is given in parentheses.

\begin{tabular}{|c|c|c|c|c|}
\hline Magnetic Laue Group & $\underline{\sigma}$ & $\underline{\sigma}^{\mathrm{x}}$ & $\underline{\sigma}^{\mathrm{y}}$ & $\underline{\sigma}^{\mathrm{z}}$ \\
\hline $\begin{array}{l}m \overline{3} m 1^{\prime} \\
(\mathrm{fcc}-\mathrm{Pt})\end{array}$ & $\left(\begin{array}{ccc}\sigma_{\mathrm{xx}} & 0 & 0 \\
0 & \sigma_{\mathrm{xx}} & 0 \\
0 & 0 & \sigma_{\mathrm{xx}}\end{array}\right)$ & $\left(\begin{array}{ccc}0 & 0 & 0 \\
0 & 0 & \sigma_{\mathrm{yz}}^{\mathrm{x}} \\
0 & -\sigma_{\mathrm{yz}}^{\mathrm{x}} & 0\end{array}\right)$ & $\left(\begin{array}{ccc}0 & 0 & -\sigma_{\mathrm{yz}}^{\mathrm{x}} \\
0 & 0 & 0 \\
\sigma_{\mathrm{yz}}^{\mathrm{x}} & 0 & 0\end{array}\right)$ & $\left(\begin{array}{ccc}0 & \sigma_{\mathrm{yz}}^{\mathrm{x}} & 0 \\
-\sigma_{\mathrm{yz}}^{\mathrm{x}} & 0 & 0 \\
0 & 0 & 0\end{array}\right)$ \\
\hline $\begin{array}{l}4 / m m^{\prime} m^{\prime} \\
\left(\text { fcc- }-\mathrm{Fe}_{x} \mathrm{Ni}_{1-x}\right)\end{array}$ & $\left(\begin{array}{ccc}\sigma_{\mathrm{xx}} & \sigma_{\mathrm{xy}} & 0 \\
-\sigma_{\mathrm{xy}} & \sigma_{\mathrm{xx}} & 0 \\
0 & 0 & \sigma_{\mathrm{zz}}\end{array}\right)$ & $\left(\begin{array}{ccc}0 & 0 & \sigma_{\mathrm{xz}}^{\mathrm{x}} \\
0 & 0 & \sigma_{\mathrm{yz}}^{\mathrm{x}} \\
\sigma_{\mathrm{zx}}^{\mathrm{x}} & \sigma_{\mathrm{zy}}^{\mathrm{x}} & 0\end{array}\right)$ & $\left(\begin{array}{ccc}0 & 0 & -\sigma_{\mathrm{yz}}^{\mathrm{x}} \\
0 & 0 & \sigma_{\mathrm{xz}}^{\mathrm{x}} \\
-\sigma_{\mathrm{zy}}^{\mathrm{x}} & \sigma_{\mathrm{zx}}^{\mathrm{x}} & 0\end{array}\right)$ & $\left(\begin{array}{ccc}\sigma_{\mathrm{xx}}^{\mathrm{z}} & \sigma_{\mathrm{xy}}^{\mathrm{z}} & 0 \\
-\sigma_{\mathrm{xy}}^{\mathrm{z}} & \sigma_{\mathrm{xx}}^{\mathrm{z}} & 0 \\
0 & 0 & \sigma_{\mathrm{zz}}^{\mathrm{z}}\end{array}\right)$ \\
\hline $\begin{array}{l}4 / m 1^{\prime} \\
\left(\mathrm{Au}_{4} \mathrm{Sc}\right)\end{array}$ & $\left(\begin{array}{ccc}\sigma_{\mathrm{xx}} & 0 & 0 \\
0 & \sigma_{\mathrm{xx}} & 0 \\
0 & 0 & \sigma_{\mathrm{zz}}\end{array}\right)$ & $\left(\begin{array}{ccc}0 & 0 & \sigma_{\mathrm{xz}}^{\mathrm{x}} \\
0 & 0 & \sigma_{\mathrm{yz}}^{\mathrm{x}} \\
\sigma_{\mathrm{zx}}^{\mathrm{x}} & \sigma_{\mathrm{zy}}^{\mathrm{x}} & 0\end{array}\right)$ & $\left(\begin{array}{ccc}0 & 0 & -\sigma_{\mathrm{yz}}^{\mathrm{x}} \\
0 & 0 & \sigma_{\mathrm{xz}}^{\mathrm{x}} \\
-\sigma_{\mathrm{zy}}^{\mathrm{x}} & \sigma_{\mathrm{zx}}^{\mathrm{x}} & 0\end{array}\right)$ & $\left(\begin{array}{ccc}\sigma_{\mathrm{xx}}^{\mathrm{z}} & \sigma_{\mathrm{xy}}^{\mathrm{z}} & 0 \\
-\sigma_{\mathrm{xy}}^{\mathrm{z}} & \sigma_{\mathrm{xx}}^{\mathrm{z}} & 0 \\
0 & 0 & \sigma_{\mathrm{zz}}^{\mathrm{z}}\end{array}\right)$ \\
\hline $\begin{array}{l}2 / m 1^{\prime} \\
\left(\mathrm{Pt}_{3} \mathrm{Ge}\right)\end{array}$ & $\left(\begin{array}{ccc}\sigma_{\mathrm{xx}} & \sigma_{\mathrm{xy}} & 0 \\
\sigma_{\mathrm{xy}} & \sigma_{\mathrm{yy}} & 0 \\
0 & 0 & \sigma_{\mathrm{zz}}\end{array}\right)$ & $\left(\begin{array}{ccc}0 & 0 & \sigma_{\mathrm{xz}}^{\mathrm{x}} \\
0 & 0 & \sigma_{\mathrm{yz}}^{\mathrm{x}} \\
\sigma_{\mathrm{zx}}^{\mathrm{x}} & \sigma_{\mathrm{zy}}^{\mathrm{x}} & 0\end{array}\right)$ & $\left(\begin{array}{ccc}0 & 0 & \sigma_{\mathrm{xz}}^{\mathrm{y}} \\
0 & 0 & \sigma_{\mathrm{yz}}^{\mathrm{y}} \\
\sigma_{\mathrm{zx}}^{\mathrm{y}} & \sigma_{\mathrm{zy}}^{\mathrm{y}} & 0\end{array}\right)$ & $\left(\begin{array}{ccc}\sigma_{\mathrm{xx}}^{\mathrm{z}} & \sigma_{\mathrm{xy}}^{\mathrm{z}} & 0 \\
\sigma_{\mathrm{yx}}^{\mathrm{z}} & \sigma_{\mathrm{yy}}^{\mathrm{z}} & 0 \\
0 & 0 & \sigma_{\mathrm{zz}}^{\mathrm{z}}\end{array}\right)$ \\
\hline
\end{tabular}

where as usual [15] $\rho$ stands for the density operator, $\beta=$ $1 / k_{B} T$ with $k_{B}$ the Boltzmann constant, $T$ is the temperature, and $\mathbf{H}$ is a magnetic field that might be present.

The shape of $\boldsymbol{\tau}$ can be found by considering the impact of a symmetry operation of the space group of the solid on Eq. (2), as this will lead to equations connecting elements of $\underline{\boldsymbol{\tau}}$. Collecting the restrictions imposed by all symmetry operations the shape of $\boldsymbol{\tau}$ is obtained. In this context it is important to note that the relevant space group of the considered system may contain not only unitary pure spatial $(u)$ but also antiunitary symmetry operations $(a)$ that involve time reversal.

The transformation properties of the operators $X=A_{i}, B_{i}$, or $C_{i}$ in Eq. (2) under symmetry operations can be expressed in terms of the corresponding Wigner D-matrices [15] $\underline{\mathbf{D}}^{(\hat{X})}(u)$ and $\underline{\mathbf{D}}^{(\hat{X})}(a)$ belonging to the operator $\hat{X}$ and the operation $u$ or $a$, respectively. Starting from Eq. (2) and making use of these transformation relations one gets the transformation behavior of $\underline{\boldsymbol{\tau}}$ under a unitary $(u)$ or antiunitary $(a)$ symmetry operation, respectively [19]:

$$
\begin{aligned}
\tau_{\left(\hat{B}_{i} \hat{C}_{j}\right) \hat{A}_{k}}(\omega, \mathbf{H})= & \sum_{l m n} \tau_{\left(\hat{B}_{m} \hat{C}_{n}\right) \hat{A}_{l}}\left(\omega, \mathbf{H}_{u}\right) \\
& \times D^{(\hat{A})}(u)_{l k} D^{(\hat{B})}(u)_{m i} D^{(\hat{C})}(u)_{n j}, \\
\tau_{\left(\hat{B}_{i} \hat{C}_{j}\right) \hat{A}_{k}}(\omega, \mathbf{H})= & \sum_{l m n} \tau_{\hat{A}_{l}^{\dagger}\left(\hat{B}_{m}^{\dagger} \hat{C}_{n}^{\dagger}\right)}\left(\omega, \mathbf{H}_{a}\right) \\
& \times D^{(\hat{A})}(a)_{l k}^{*} D^{(\hat{B})}(a)_{m i}^{*} D^{(\hat{C})}(a)_{n j}^{*} .
\end{aligned}
$$

It should be noted that in general the tensors $\tau_{\left(\hat{B}_{i} \hat{C}_{j}\right) \hat{A}_{k}}$ and $\tau_{\hat{A}_{k}^{\dagger}\left(\hat{B}_{i}^{\dagger} \hat{C}_{j}^{\dagger}\right)}$ are different objects representing different response functions which are only interrelated by Eq. (4). It nevertheless imposes restrictions on the shape of $\tau_{\left(\hat{B}_{i} \hat{C}_{j}\right) \hat{A}_{k}}$ giving rise to (generalized) Onsager relations.

Assuming $\hat{C}_{i}=1$ and $\hat{B}_{i}=\hat{A}_{i}=\hat{j}_{i}$ with $\hat{j}_{i}$ the current density operator $\underline{\tau}$ corresponds to the ordinary electrical conductivity tensor $\underline{\sigma}$. Using the behavior of $\hat{j}_{i}$ under symmetry operations [15], it turns out that only the magnetic Laue group of the system has to be considered, that is generated by adding the (space) inversion operation $I$ to the crystallographic magnetic point group [20]. The resulting shape of the conductivity tensor $\boldsymbol{\sigma}$ is given in Table I for four different magnetic Laue groups [19].

When considering the spin conductivity tensor its elements $\sigma_{i j}^{k}$ give the current density along direction $i$ for the spin polarization with respect to the $k$ axis induced by an electrical field along the $j$ axis. In this case the perturbing electric field is still represented by $\hat{A}_{i}=\hat{j}_{i}$ while the induced spin current density is represented by the corresponding operator $\hat{J}_{i}^{k}=\left(\hat{B}_{i} \hat{C}_{k}\right)$. As the explicit definition of $\hat{J}_{i}^{k}$ is not relevant for the following, but only its symmetry properties, the frequently used nonrelativistic definition $\hat{J}_{i}^{k}=\frac{1}{2}\left\{\hat{v}_{i}, \sigma_{k}\right\}$ may be used that consists of a combination of the Pauli spin matrix $\sigma_{k}$ and the conventional velocity operator $\hat{v}_{i}$ [21]. Alternatively, one may use the relativistic definition of the spin current operator $\hat{J}_{i}^{k}=\hat{\mathcal{T}}_{k} \hat{j}_{i}$ as suggested by Vernes et al. [22] that involves the spatial part $\hat{\mathcal{T}}_{k}$ of the spin polarization operator [23].

Expressing the transformation behavior of $\hat{J}_{i}^{k}$ in terms of the Wigner matrices allows deducing the shape of the corresponding spin conductivity tensor on the basis of Eqs. (3) and (4). As for the electrical conductivity it turns out again that one has to consider only the magnetic Laue group; i.e., there are only 37 different cases. Table I gives for the four cases considered here the shape of the various subtensors $\underline{\sigma}^{k}$, where $k$ specifies the component of the spin polarization.

Considering a nonmagnetic metal with fcc or bcc structure $\left(m \overline{3} m 1^{\prime}\right)$ Kleiner's scheme naturally leads to an isotropic electrical conductivity tensor $\underline{\sigma}$. The extension to deal with the spin conductivity tensor sketched above gives in this case only a few nonvanishing elements that are associated with the SHE and are symmetry related according to $\sigma_{\mathrm{yz}}^{\mathrm{x}}=$ $\sigma_{\mathrm{zx}}^{\mathrm{y}}=\sigma_{\mathrm{xy}}^{\mathrm{z}}=-\sigma_{\mathrm{zy}}^{\mathrm{x}}=-\sigma_{\mathrm{xz}}^{\mathrm{y}}=-\sigma_{\mathrm{yx}}^{\mathrm{z}}$; i.e., cyclic permutation of the indices gives no change while anticyclic permutation changes the sign. In contrast to other derivations, there is obviously no need to artificially introduce a spin-projected conductivity or to make reference to the conductivity tensor of a spin-polarized solid. For a ferromagnetic metal with fcc or bcc structure $\left(4 / \mathrm{mm}^{\prime} \mathrm{m}^{\prime}\right)$ with the magnetization along the $\mathrm{z}$ direction, the well-known shape of the conductivity tensor $\boldsymbol{\sigma}$ is obtained that reflects the anomalous Hall effect $\left(\sigma_{\mathrm{xy}}\right)$ as well as the magnetoresistance anisotropy $\left(\sigma_{\mathrm{xx}} \neq \sigma_{\mathrm{zz}}\right)$ with the symmetry relations $\sigma_{\mathrm{xy}}=-\sigma_{\mathrm{yx}}$ and $\sigma_{\mathrm{xx}}=\sigma_{\mathrm{yy}}$. The 
spin conductivity tensor $\sigma^{z}$ shows as for the nonmagnetic case antisymmetric off-diagonal elements that represent the transverse spin conductivity. This implies the occurrence of the spin Hall effect in ferromagnets that was investigated recently for diluted alloys [24]. For polarization along the $\mathrm{x}$ and $\mathrm{y}$ axes, however, different although still interrelated elements appear as compared to the nonmagnetic case since fewer symmetry relations survive in the presence of a spontaneous magnetization. Additionally, in contrast to the nonmagnetic case also a longitudinal spin-polarized conductivity $\left(\sigma_{i i}^{\mathrm{Z}}\right)$ occurs in a ferromagnet, that for example gives rise to the spin-dependent Seebeck effect [25]. A simple explanation for the corresponding longitudinal spin transport would be based on Mott's two-current model assuming different conductivities for the two spin channels. However, it is well known that spin-orbit interaction leads to a hybridization of the spin channels and influences even the longitudinal conductivity of a ferromagnet this way [26]. Accordingly, it cannot be ruled out that the longitudinal tensor elements $\sigma_{i i}^{\mathrm{Z}}$ are not only reflecting the spontaneous spin magnetization of the material but are to some extent due to spin-orbit coupling.

Indeed the scheme presented above leads for nonmagnetic systems having low symmetry not only to off-diagonal elements reflecting transverse spin conductivity, i.e., the SHE, but also to diagonal elements reflecting longitudinal spin transport, that was not observed so far. For the two magnetic Laue groups $4 / m 1^{\prime}$ and $2 / m 1^{\prime}$ for nonmagnetic solids considered in Table I, a 4- and 2-fold, resp., rotation axis is present. As a consequence longitudinal spin currents show up only with spin polarization along this principal axis of rotation.

To verify the results of our group-theoretical approach independently we calculated the full spin conductivity tensor for solids having different structures corresponding to different magnetic Laue groups. This work employs a computational scheme that has been used before for numerical studies on the SHE in nonmagnetic transition metal alloys [27]. Performing these calculations without making use of symmetry led numerically to a spin conductivity tensor that was always fully in line with the analytical group-theoretical results concerning the shape and degeneracies of the tensor.

To get a first estimate of the order of magnitude of the longitudinal spin-polarized conductivity in nonmagnets, calculations have been done for the system $\left(\mathrm{Au}_{1-x} \mathrm{Pt}_{x}\right)_{4} \mathrm{Sc}$ having the magnetic Laue group 4/m $1^{\prime}$ for varying Pt concentration $x$. Figure 1 (top) shows the corresponding electrical conductivity that is, in agreement with Table I, diagonal and slightly anisotropic; i.e., $\sigma_{\mathrm{xx}}=\sigma_{\mathrm{yy}} \approx \sigma_{\mathrm{zz}}$.

Furthermore, the conductivities $\sigma_{i i}$ are strongly asymmetric with respect to the concentration $x$ when replacing Au with prominent $s p$ character at the Fermi level by Pt with dominant $d$ character. Furthermore, one notes a relatively strong impact of the vertex corrections on the Au-rich side of the system $(x \approx$ $0)$ while these are much less important on the Au-poor side $(x \approx 1)$. This observation is well known from binary transition metal alloys, such as $\mathrm{Cu}_{1-x} \mathrm{Pt}_{x}$ [28] or $\mathrm{Ag}_{1-x} \mathrm{Pd}_{x}$ [29], where the dominance of $s p$ character changes to $d$ character when $x$ is varied from 0 to 1 .

The transverse spin conductivity $\sigma_{i j}^{\mathrm{x}}$ is shown in the middle panel of Fig. 1 for $\mathrm{x}$ polarization of the spin. As Table I shows going from $m \overline{3} m 1^{\prime}$ to $4 / m 1^{\prime}$ symmetry the relation

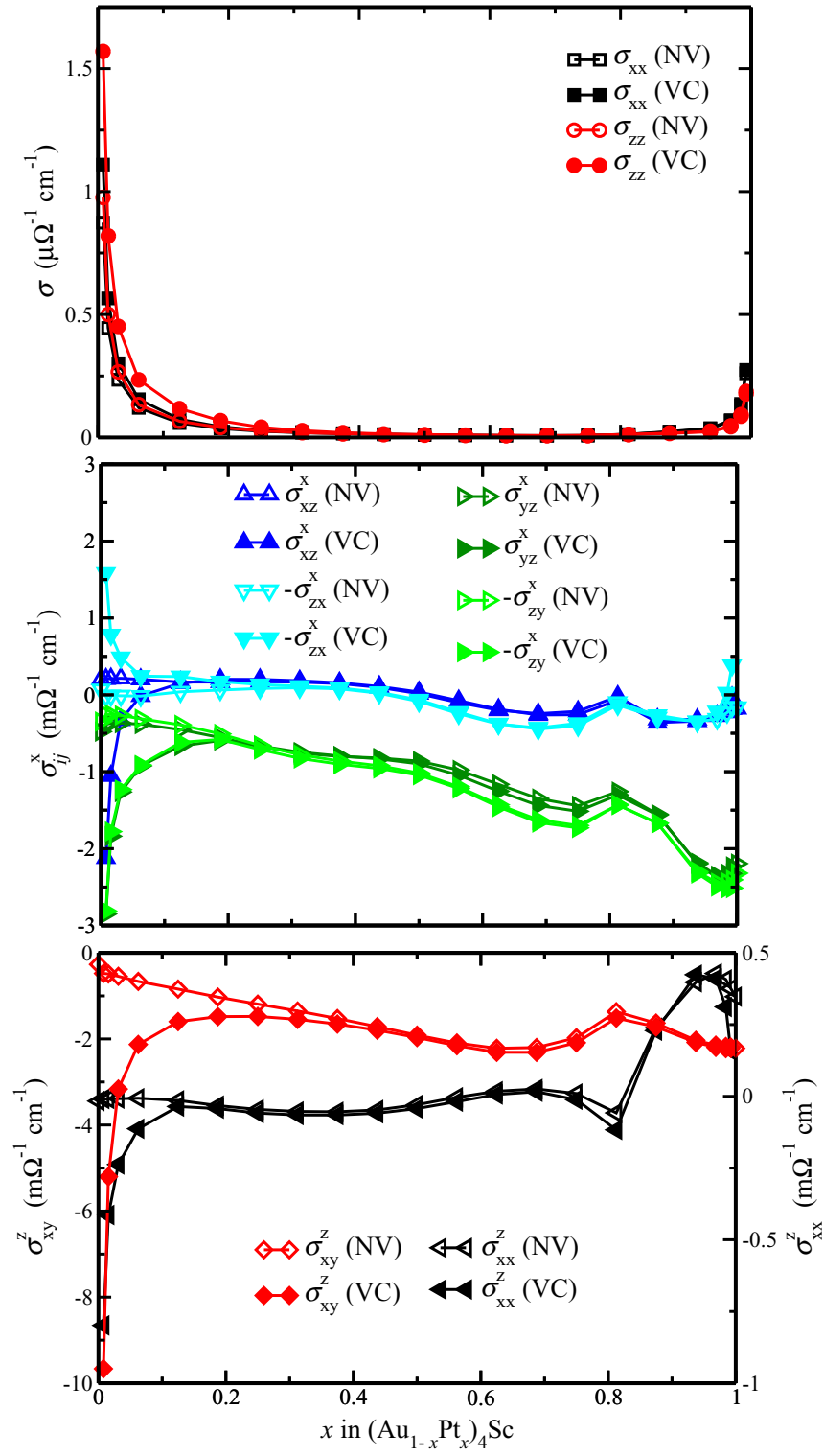

FIG. 1. (Color online) Top: Longitudinal conductivity $\sigma_{i i}$ for $\left(\mathrm{Au}_{1-x} \mathrm{Pt}_{x}\right)_{4} \mathrm{Sc}$ as a function of the concentration $x$ calculated without $(\mathrm{NV})$ and with (VC) the vertex corrections. Middle: Transverse spin conductivities $\sigma_{i j}^{\mathrm{x}}$. Bottom: Transverse and longitudinal spin conductivity $\sigma_{\mathrm{xy}}^{\mathrm{z}}$ and $\sigma_{\mathrm{xx}}^{\mathrm{z}}$, respectively.

$\sigma_{\mathrm{yz}}^{\mathrm{x}}=-\sigma_{\mathrm{zy}}^{\mathrm{x}}$ disappears; i.e., the corresponding subtensor is not antisymmetric anymore. A symmetric component, which is by definition not present in the ordinary SHE, indeed can be seen in Fig. 1 (middle) although the deviations are not very pronounced. In line one finds (except for $x \rightarrow 0$ ) for the additional nonzero tensor elements $\sigma_{\mathrm{xz}}^{\mathrm{x}} \approx-\sigma_{\mathrm{zx}}^{\mathrm{x}}$. The first coefficient relates a spin current $j_{\mathrm{x}}^{\mathrm{x}}$ polarized in the direction of motion to an electric field $E_{\mathrm{z}}$, whereas $\sigma_{\mathrm{zx}}^{\mathrm{x}}$ describes a spin current $j_{\mathrm{z}}^{\mathrm{x}}$ transverse, but with the spin polarization parallel to the driving electric field $E_{\mathrm{x}}$. To our knowledge the corresponding effects have not been considered so far. Interestingly, both elements occur simultaneously for a given magnetic Laue group or both are absent. However, compared to the spin-Hall-like elements $\sigma_{\mathrm{yz}}^{\mathrm{x}}$ and $\sigma_{\mathrm{zy}}^{\mathrm{x}}$ they are smaller. For 

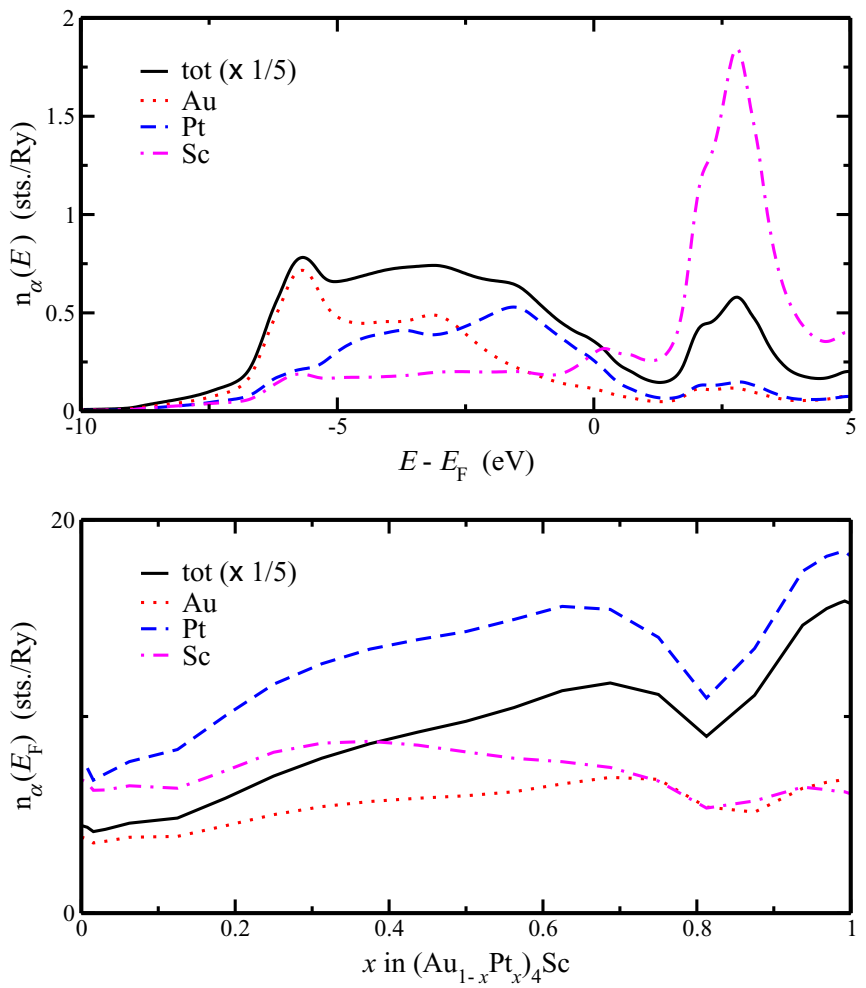

FIG. 2. (Color online) Top: Energy-dependent component- $(\alpha)$ resolved DOS $n_{\alpha}(E)$ for $\left(\mathrm{Au}_{0.5} \mathrm{Pt}_{0.5}\right)_{4} \mathrm{Sc}$. Bottom: Componentresolved DOS $n_{\alpha}\left(E_{\mathrm{F}}\right)$ at the Fermi energy $E_{\mathrm{F}}$ for $\left(\mathrm{Au}_{1-x} \mathrm{Pt}_{x}\right)_{4} \mathrm{Sc}$ as a function of the concentration $x$.

$y$ polarization of the spin the corresponding tensor elements are uniquely related to those for $\mathrm{x}$ polarization according to Table I and for this reason not given here. The tensor elements $\sigma_{i j}^{\mathrm{z}}$ for $\mathrm{z}$ polarization are given in the lower panel of Fig. 1. In line with Table I they obey the symmetry relation $\sigma_{\mathrm{xy}}^{\mathrm{z}}=-\sigma_{\mathrm{yx}}^{\mathrm{z}}$ (i.e., describing the pure SHE) and differ from $\sigma_{\mathrm{yz}}^{\mathrm{x}}$. This difference however is, except again for $x \rightarrow 0$, not very pronounced. In particular, $\sigma_{\mathrm{yz}}^{\mathrm{x}}$ and $\sigma_{\mathrm{xy}}^{\mathrm{z}}$ show a similar variation with concentration $x$ that differs clearly from that of the longitudinal spin conductivity $\sigma_{\mathrm{xx}}^{\mathrm{z}}$ shown as well in Fig. 1 (bottom). Although this new type of tensor element is overall somewhat smaller in magnitude than the dominating transverse elements it has nevertheless the same order of magnitude, especially in the Au-rich regime, and for that reason it should be possible to determine it experimentally.

As can be seen in Fig. 1 the curves for the spin conductivity tensor elements $\sigma_{i j}^{k}$ as function of the concentration $x$ are much more structured than the electrical conductivity $\sigma_{i i}$; i.e., they are much more strongly affected by the variation of the electronic structure with composition. In particular the spin conductivities $\sigma_{i j}^{k}$ show pronounced peaks or dips for $x \approx 0.8$. This behavior can be related to the variation of the density of states (DOS) with $x$ as can be seen from Fig. 2. The figure shows the component-resolved DOS $n_{\alpha}(E)$ as a function of the energy $E$ for $\left(\mathrm{Au}_{0.5} \mathrm{Pt}_{0.5}\right)_{4} \mathrm{Sc}$ (top) and at the Fermi energy $E_{\mathrm{F}}$ for $\left(\mathrm{Au}_{1-x} \mathrm{Pt}_{x}\right)_{4} \mathrm{Sc}$ as a function of the concentration $x$ (bottom). As mentioned above, at the Fermi energy the partial DOS $n_{\mathrm{Au}}\left(E_{\mathrm{F}}\right)$ of $\mathrm{Au}$ is dominated by $s p$ states while that of $\mathrm{Pt}$ has dominant $d$ character. The pronounced dip of the Pt DOS $n_{\mathrm{Pt}}\left(E_{\mathrm{F}}\right)$ at $x \approx 0.8$ is apparently responsible for the prominent features in the spin conductivity curves shown in Fig. 1 (middle and bottom panels). As mentioned before, for the longitudinal conductivity $\sigma_{i i}$ inclusion of the vertex corrections has primarily an impact at the Au-rich side of the system. The same behavior is found for the transverse $\left(\sigma_{i j}^{k}\right)$ as well as the longitudinal $\left(\sigma_{i i}^{\mathrm{Z}}\right)$ spin conductivity components. For the transverse spin Hall conductivity it could be demonstrated that the contribution connected with the vertex corrections corresponds to the so-called extrinsic contribution that is primarily caused by the skew scattering mechanism $[24,27]$. The very similar dependence of $\sigma_{i j}^{k}$ and $\sigma_{i i}^{\mathrm{z}}$ on the vertex corrections suggests that this applies also for the longitudinal spin conductivity.

In summary, a group-theoretical scheme has been presented that allows determining the shape of response tensors relevant for the field of spintronics. Application to the spin conductivity tensor gave a sound and model-independent explanation for the occurrence of the transverse tensor elements responsible for the spin Hall effect and two additional, closely related effects. In addition it was found that for low symmetry longitudinal elements show up in addition even for nonmagnetic solids that were not considered before. Independent numerical investigations confirmed these results and demonstrated for $\left(\mathrm{Au}_{1-x} \mathrm{Pt}_{x}\right)_{4} \mathrm{Sc}$ that the longitudinal spin conductivity may be on the same order of magnitude as the transverse one. It should be noted that the discussion of the spin conductivity tensor was referring to the dc limit $\omega=0$. However, the tensor forms given in Table I also hold for finite frequencies, implying the occurrence of the ac counterparts to the discussed effects. In addition, the formalism is applicable to numerous other linear response phenomena as, e.g., the AHE, anisotropic magnetoresistance (AMR), the Edelstein effect [4,5], Gilbert damping [30], spin-orbit torques [31], etc. Furthermore, using the fact that the operators for electrical and heat currents share the same transformation properties the presented formalism can be applied to spincaloritronic phenomena as well.

This work was supported financially by the Deutsche Forschungsgemeinschaft (DFG) under the priority program SPP 1538 (Spin Caloric Transport) and the SFB 689 (Spinphänomene in reduzierten Dimensionen). Discussions with $\mathrm{Ch}$. Back and $\mathrm{H}$. Hübl are gratefully acknowledged.
[1] M. Dyakonov and V. Perel, Phys. Lett. A 35, 459 (1971).

[2] J. E. Hirsch, Phys. Rev. Lett. 83, 1834 (1999).

[3] J. Sinova, D. Culcer, Q. Niu, N. A. Sinitsyn, T. Jungwirth, and A. H. MacDonald, Phys. Rev. Lett. 92, 126603 (2004).
[4] A. G. Aronov and Y. B. Lyanda-Geller, Zh. Eksp. Teor. Fiz. 50, 398 (1989) [JETP Lett. 50, 431 (1989)].

[5] V. M. Edelstein, Solid State Commun. 73, 233 (1990).

[6] K. Tauber, M. Gradhand, D. V. Fedorov, and I. Mertig, Phys. Rev. Lett. 109, 026601 (2012). 
[7] S. Wimmer, D. Ködderitzsch, K. Chadova, and H. Ebert, Phys. Rev. B 88, 201108 (2013).

[8] J. Weischenberg, F. Freimuth, S. Blügel, and Y. Mokrousov, Phys. Rev. B 87, 060406 (2013).

[9] S. Wimmer, D. Ködderitzsch, and H. Ebert, Phys. Rev. B 89, 161101 (2014).

[10] Z. Fang et al., Science 302, 92 (2003).

[11] Y. Yao and Z. Fang, Phys. Rev. Lett. 95, 156601 (2005).

[12] N. Nagaosa, J. Sinova, S. Onoda, A. H. MacDonald, and N. P. Ong, Rev. Mod. Phys. 82, 1539 (2010).

[13] N. A. Sinitsyn, J. Phys.: Condens. Matter 20, 023201 (2008).

[14] M. Gradhand, D. V. Fedorov, P. Zahn, and I. Mertig, Phys. Rev. Lett. 104, 186403 (2010).

[15] W. H. Kleiner, Phys. Rev. 142, 318 (1966).

[16] W. H. Kleiner, Phys. Rev. 153, 726 (1967).

[17] More precisely, one should use the expression spin polarization current density operator, but we keep the abbreviated term also in the relativistic context.

[18] M. Seemann, D. Ködderitzsch, S. Wimmer, and H. Ebert (unpublished).

[19] See Supplemental Material at http://link.aps.org/supplemental/ 10.1103/PhysRevB.92.041101 for a sketch of the derivation of Eq. (3), notes on the application of Eqs. (3) and (4) to the spinpolarized conductivity tensor, including results for all possible magnetic Laue groups of nonmagnetic solids, and details of the first-principles approach used.

[20] In contrast to Kleiner we adopt here the definition of a Laue group that is in general use nowadays.

[21] J. Shi, P. Zhang, D. Xiao, and Q. Niu, Phys. Rev. Lett. 96, 076604 (2006).

[22] A. Vernes, B. L. Györffy, and P. Weinberger, Phys. Rev. B 76, 012408 (2007).

[23] V. Bargmann and E. P. Wigner, Proc. Natl. Acad. Sci. USA 34, 211 (1948).

[24] B. Zimmermann et al., Phys. Rev. B 90, 220403 (2014).

[25] A. Slachter, F. L. Bakker, J.-P. Adam, and B. J. van Wees, Nat. Phys. 6, 879 (2010).

[26] J. Banhart, H. Ebert, and A. Vernes, Phys. Rev. B 56, 10165 (1997).

[27] S. Lowitzer, M. Gradhand, D. Ködderitzsch, D. V. Fedorov, I. Mertig, and H. Ebert, Phys. Rev. Lett. 106, 056601 (2011).

[28] J. Banhart, H. Ebert, P. Weinberger, and J. Voitländer, Phys. Rev. B 50, 2104 (1994).

[29] P. R. Tulip, J. B. Staunton, S. Lowitzer, D. Ködderitzsch, and H. Ebert, Phys. Rev. B 77, 165116 (2008).

[30] H. Ebert, S. Mankovsky, D. Ködderitzsch, and P. J. Kelly, Phys. Rev. Lett. 107, 066603 (2011).

[31] K. Garello et al., Nat. Nanotechnol. 8, 587 (2013). 